\title{
Inflammation-regulating factors in ascites as predictive biomarkers of drug resistance and progression-free survival in serous epithelial ovarian cancers
}

Denis Lane ${ }^{1}$, Isabelle Matte ${ }^{1}$, Perrine Garde-Granger², Claude Laplante², Alex Carignan' ${ }^{1}$, Claudine Rancourt ${ }^{1}$ and Alain Piché ${ }^{*}$

\begin{abstract}
Background: Platinum-based combination therapy is the standard first-line treatment for women with advanced serous epithelial ovarian carcinoma (EOC). However, about $20 \%$ will not respond and are considered clinically resistant. The availability of biomarkers to predict responses to the initial therapy would provide a practical approach to identify women who would benefit from a more appropriate first-line treatment. Ascites is an attractive inflammatory fluid for biomarker discovery as it is easy and minimally invasive to obtain. The aim of this study was to evaluate whether six selected inflammation-regulating factors in ascites could serve as diagnostic or drug resistance biomarkers in patients with advanced serous EOC.
\end{abstract}

Methods: A total of 53 women with stage III/IV serous EOC and 10 women with benign conditions were enrolled in this study. Eleven of the 53 women with serous EOC were considered clinically resistant to treatment with progression-free survival $<6$ months. Ascites were collected at the time of the debulking surgery and the levels of cytokines were measured by ELISA. The six selected cytokines were evaluated for their ability to discriminate serous EOC from benign controls, and to discriminate platinum resistant from platinum sensitive patients.

Results: Median ascites levels of IL-6, IL-10 and osteoprotegerin (OPG) were significantly higher in women with advanced serous EOC than in controls $(P \leq 0.012)$. There were no significant difference in the median ascites levels of leptin, soluble urokinase plasminogen activator receptor (suPAR) and CCL18 among serous EOC women and controls. In Receiver Operator curve (ROC) analysis, IL-6, IL-10 and OPG had a high area under the curve value of $0.905,0.832$ and 0.825 respectively for distinguishing EOC from benign controls. ROC analysis of individual cytokines revealed low discriminating potential to stratify patients according to their sensitivity to first-line treatment. The combination of biomarkers with the highest discriminating potential was with CA125 and leptin ( $A \cup C=0.936,95 \% \mathrm{Cl}: 0.894-0.978)$.

Conclusion: IL- 6 was found to be strongly associated with advanced serous EOC and could be used in combination with serum CA125 to discriminate benign and EOC. Furthermore, the combination of serum CA125 and ascites leptin was a strong predictor of clinical resistance to first-line therapy.

Keywords: Ascites, Ovarian cancer, Tumor microenvironment, Cytokines, Inflammation, Drug resistance

\footnotetext{
* Correspondence: alain.piche@usherbrooke.ca

'Département de Microbiologie et Infectiologie, Faculté de Médecine,

Université de Sherbrooke, 3001, 12ième Avenue Nord, J1H 5 N4 Sherbrooke,

Canada

Full list of author information is available at the end of the article
}

\section{Biomed Central}

(c) 2015 Lane et al. This is an Open Access article distributed under the terms of the Creative Commons Attribution License (http://creativecommons.org/licenses/by/4.0), which permits unrestricted use, distribution, and reproduction in any medium, provided the original work is properly credited. The Creative Commons Public Domain Dedication waiver (http:// creativecommons.org/publicdomain/zero/1.0/) applies to the data made available in this article, unless otherwise stated. 


\section{Background}

Epithelial ovarian cancer (EOC) is the leading cause of gynecological cancer-related death $[1,2]$. Serous carcinomas are the most frequent subtype encountered in patients with EOC [3]. Being largely asymptomatic, over $70 \%$ of patients are diagnosed at an advanced stage of the disease (stage III/IV) with metastasis throughout the peritoneal cavity and large amount of ascites [1, 3, 4]. Platinum-based combination chemotherapy is the standard first-line treatement for advanced stage EOC. Although overall initial response rates to first-line platinum based chemotherapy are good, $15-20 \%$ of patients will not respond to the initial chemotherapy [5]. The tumors are considered resistant if the patient do not respond to platinum-based therapy or show progression during the course of therapy, or if the clinical progression-free survival (PFS) is less than 6 months [6]. These patients are considered to have intrinsic resistance to first-line treatment. There is currently no available biomarker to identify these patients at baseline. Unfortunately, these patients are identified retrospectively after they experienced early relapse or did not respond to initial treatment. Thus, customised treatments and clinical stratification of these EOC patient remain critical objectives in the field. The identification of new biomarkers for intrinsic drug resistance would represent a substantial step forward in our efforts to adequately treat EOC and increase survival.

The only clinically validated biomarker for disease monitoring and assessing response and relapse to treatment is CA125 which is encoded by MUC16 mucin gene [7-12]. The N-terminal extracellular region of MUC16 is cleaved and released into the serum of patients with EOC [9]. Serum CA125 lacks specificity and sensitivity, as a single marker, for early EOC detection and prognosis [13]. Recent studies suggest that a Risk of Ovarian Malignancy Algorithm (ROMA) incorporating CA125 and HE4 levels in serum shows a high potential for discriminating ovarian cancer from benign gynecological diseases [14-16]. HE4 is the only biomarker, other than CA125, that has been approved as a diagnostic marker for ovarian cancer [17].

Tumor-promoting inflammation is now established as a hallmark of cancer $[18,19]$. Serum cytokine levels have been investigated as diagnostic and prognostic markers in ovarian cancer. Ascites from women with advanced serous EOC is an inflammatory milieu rich in inflammation promoting factors. An inflammatory environment such as ascites promotes drug resistance of EOC cells [20-23]. High levels of pro-inflammatory cytokines, chemokines and growth factors are found in OC ascites [23-29]. A recent multiplex profiling of cytokines in the ascites of 10 EOC patients has demonstrated enhanced expression of several inflammation-regulating factors including IL-6, IL-6R, IL-8, IL-10, leptin, osteoprotegerin (OPG) and urokinase plasminogen activator (uPAR) among others [30]. Specific inflammatory cytokines in ascites such as IL-6 were shown to be an independent prognostic factor of worse outcome [31]. IL-6 contributes to EOC progression by inhibition of apoptosis, stimulation of angiogenesis, increased migration and invasion, and stimulation of cell proliferation [32-35].

Ascites is an attractive biofluid for biomarker discovery as it is easy and minimally invasive to obtain. Proximal fluids such as ascites - as opposed to serum - might reflect events in ovarian tumorigenesis earlier than in peripheral blood circulation [36]. Furthermore, the concentration of cytokines is usually much higher in ascites compared to serum [29]. Thus, the accessibility of ascites - a simple non-invasive puncture - provides an excellent source of inflammation promoting factors (with potential enrichment relative to serum) for the investigation of prognostic biomarkers.

Ascites from a small subset of serous EOC patients and patients with benign gynecological conditions has been previously analyzed with a panel of 120 cytokines by cytokine array [30]. This analysis has revealed 20 cytokines/growth factors, which showed a statistically significant $(P<0.01)>2$-fold up-regulation relative to benign fluids. For this study, six inflammatory-regulating factors including IL-6, IL-10, leptin, osteoprotegerin (OPG), soluble urokinase plasminogen activator receptor (suPAR) and CCL18 were initially selected based on the following biological rationales: 1) IL-6, IL-10, leptin, OPG, suPAR and CCL18 are present at high levels in EOC ascites [29, 30]; 2) high ascites levels of IL-6, IL-10, leptin and OPG have been associated with EOC worse outcome [30]; 3) their concentrations in ascites are well within the range required to induce a biological effect $[29,30]$; 4) IL-6, IL10, leptin, suPAR and OPG can inhibit drug-induced apoptosis in vitro in EOC cells or other cancer cells [34, 37-46].

In the present study, we have measured the baseline levels of six inflammation-regulating factors including IL-6, IL-10, leptin, OPG, suPAR and CCL18 in prospectively collected ascites patients with advanced serous EOC with complete clinicopathologic data and adequate follow up. The aims of the study was to establish (1) whether levels of these cytokines differ between benign and serous EOC, (2) whether levels can distinct patients with intrinsic drug resistance to those that respond to first-line platinum-based treatment.

\section{Methods}

\section{Patients}

Ascites is routinely obtained at the time of the debulking surgery of ovarian cancer patients treated at the Centre Hospitalier Universitaire de Sherbrooke. After collection, 
cell-free ascites are stored at $-80{ }^{\circ} \mathrm{C}$ in our tumor bank until use. The study population consisted of 53 women with newly diagnosed epithelial ovarian cancer admitted at the Centre Hospitalier Universitaire de Sherbrooke. Ten cases with benign conditions, namely histologically benign gynecological conditions including fibromas (5), mucinous and serous cystadenomas (4), and one inflammatory lesion, constituted the control group. This study was approved by the Institutional Review Board of the Centre de Recherche Étienne-Le Bel. Informed consent was obtained from women that underwent surgery by the gynecologic oncology service between 2000 and 2013. All samples were reviewed by an experienced pathologist. Baseline characteristics and serum CA125 levels were collected for all patients. All patients had a follow up $\geq 12$ months. Disease progression was defined by either serum CA125 $\geq 2 \mathrm{X}$ nadir value on two occasions, documentation of lesion progression or appearance of new lesions on CT-scan or death [37]. Patient's conditions were staged according to the criteria of the International Federation of Gynecology and Obstetrics (FIGO). PFS was defined by the time from the initial surgery to evidence of disease progression. Drug resistance was defined as those with PFS $<6$ months or lack of response to initial platinum-based chemotherapy. Patient characteristics are summarised in Table 2.

\section{Peritoneal fluid specimens}

Peritoneal fluids and ascites were obtained at the time of initial cytoreductive surgery for all patients. Peritoneal fluids were centrifuged at $1000 \mathrm{rpm}$ for $15 \mathrm{~min}$ and cellfree supernatants were stored at $-80{ }^{\circ} \mathrm{C}$ until assayed. All acellular fluids were supplied by the Banque de tissus et de données of the Réseau de Recherche en Cancer of the Fonds de la Recherche du Québec en Santé affiliated to the Canadian Tumor Repository Network (CTRNet).

\section{ELISA measurements}

Cytokine levels in peritoneal fluid samples were determined by ELISA using the commercially available human Quantikine kits from R\&D Systems (Minneapolis, MN).
OPG levels were determined using an ELISA from $\mathrm{E}$ Bioscience (Vienna, Austria). The assays were performed in duplicate according to the manufacturer's protocols. The detection thresholds were $0.79 \mathrm{pg} / \mathrm{ml}$ for IL-6, $2.9 \mathrm{pg} /$ $\mathrm{ml}$ for IL-10, $7.8 \mathrm{pg} / \mathrm{ml}$ for leptin, $4.5 \mathrm{pg} / \mathrm{ml}$ for OPG, $33 \mathrm{pg} / \mathrm{ml}$ for suPAR and $1.1 \mathrm{ng} / \mathrm{ml}$ for CCL18. The intraassay variability was $5-10 \%$ for IL-6, $2.5-6.6 \%$ for IL-10, 3-3.2 \% for leptin, $4.3-7.9 \%$ for OPG, $2.1-7.5 \%$ for suPAR and $3.2-3.7 \%$ for CCL18. The inter-assay variability varied from 3.5 to $7.6 \%$ depending on the cytokine. All samples were examined in duplicate and the median values were used for statistical analysis.

\section{CA125 measurements}

CA125 was determined at Centre Hospitalier Universitaire de Sherbrooke laboratory in serum samples by EIA using the Elecsys 2010 analyzer and CA125 II regents (Roche Diagnostics, Québec, Canada). The reference range was $0-35 \mathrm{kUI} / \mathrm{L}$.

\section{Statistical analysis}

Comparison between unpaired groups was made using the Mann-Whitney test or the Kruskal-Wallis test. Statistical differences in PFS were determined by the log-rank test, and Kaplan-Meier survival curves were made. PFS was defined as the interval between the date of the initial debulking surgery and the time of disease progression or the last date of follow up. Receiveroperator curves (ROC) were created to determine the predictive value of the cytokines to distinguish between EOC patients and control, and between clinically resistant and sensitive patients. The threshold for statistical significance is $P<0.05$.

\section{Results}

Predictive value of ascites inflammation-regulating factors for EOC versus control group

Expression levels of IL-6, IL-10, leptin, OPG, suPAR and CCL18 in ascites were measured by ELISA. These inflammation-regulating factors were measured in a cohort of 53 patients with advanced (stage III/IV) serous EOC

Table 1 Ascites levels of the selected inflammatory cytokines

\begin{tabular}{lllll}
\hline Cytokines & Benign controls median, pg/ml & Serous EOC median, pg/ml & Fold change (FC) relative to benign & $P$ value \\
\hline IL-6 & $15(6-65)$ & $1820(279-4327)$ & 121 & $<0.001$ \\
IL-10 & $10(0-45)$ & $97,5(23-186)$ & 9.8 & $<0.001$ \\
Leptin & $254(152-917)$ & $453(177-1956)$ & 1.8 & NS \\
SUPAR & $272(89-15,944)$ & $7021(1170-15,538)$ & 28.8 & NS \\
CCL18 & $15,000(2000-27,000)$ & $20,000(3000-39,000)$ & 1.3 & NS \\
OPG & $18(2-166)$ & $296(23-865)$ & 16,4 & 0.012 \\
\hline
\end{tabular}

Values in brackets indicate $25-75$ quartiles

NS not statistically significant

$P$ value $=$ Student $T$ test 


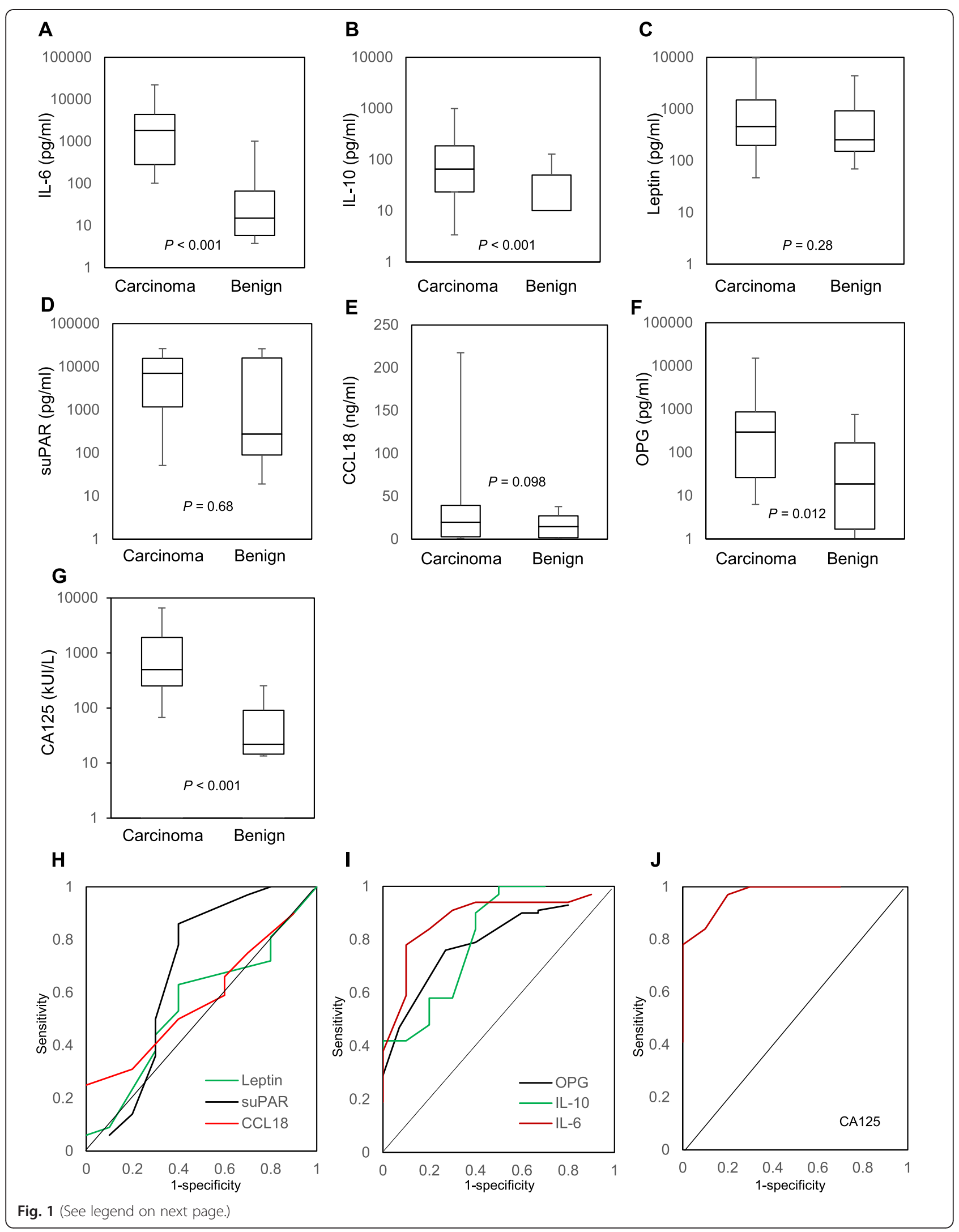


(See figure on previous page.)

Fig. 1 Ascites levels of inflammation-regulating factors in serous EOC patients and those with benign conditions. Box plots representing ascites levels of IL-6 (a), IL-10 (b), leptin (c), suPAR (d), CCL18 (e) and OPG (f) in patients with advanced serous EOC and patients with benign gynecological conditions. (g) Box plot of serum CA125 levels in serous EOC patients and patients with benign gynecological diseases. The $P$ value is indicated for each factor. ROC analysis using leptin, suPAR and CCL18 (h), and IL-6, IL-10 and OPG (i) for distinguishing patients with serous EOC from control patients. (j) ROC analysis of serum CA125 for distinguishing serous EOC from control patients

from ascites that were obtained at the time of their debulking surgery. Median IL-6 ascites levels were 121-fold, IL-10 levels 9.8-fold and OPG levels 16.4-fold higher in serous EOC samples compared to benign controls (Table 1, Fig. 1a, b and f). In contrast, median CCL18 and leptin ascites levels were not statistically different in serous EOC compared to benign controls (Table 1, Fig. 1c and e). Although, median levels of suPAR were almost 29-fold higher in serous EOC patients, the difference was not statistically significant $(P=0.68)$ (Table 1 , Fig. 1d). IL-6 and IL-10 levels were undetectable in $6 \%$ of serous EOC and in $10 \%$ and $40 \%$ of the benign controls respectively. Serum CA125 levels were measured and the median level was 23-fold higher in serous EOC sample compared to control with a $P<0.001$ (Fig. 1g). The expression of IL-6 in the ascites of serous EOC patients did not show a strong correlation with those of IL-10 (correlation coefficient, $R<0.1$ ). We also observed a lack of significant correlation between the expression of IL-6 and those of leptin, suPAR and CCL18 with $R<0.1$.

ROC analyses were performed to determine the predictive value of ascites factors distinguishing EOC patients from the control group. Ascites levels of IL-6 allowed most accurate discrimination (AUC $=0.905$, 95 \% CI: 0.850-0.960) between EOC patients and benign controls although it did not outperformed serum CA125 (AUC $=0.951,95$ \% CI: 0.906-0.996) (Fig. 1i and j). IL10 and OPG also discriminated serous EOC patients from benign controls with AUC $=0.832$ (95 \% CI: 0.7630.901 ) and $\mathrm{AUC}=0.825$ (95\% CI: 0.782-0.868 respectively (Fig. 1j). The other inflammation-regulating factors tested had lower discriminating potential with AUC for suPAR $=0.757$ (95\% CI: 0.632-0.882), for leptin $=0.586$
(95 \% CI: 0.488-0.684) and for CCL18 = 0.612 (95\% CI: 0.538-0.686) (Fig. 1h). The results did not reach statistical significance for suPAR, leptin and CCL18. Thus, ascites levels of IL-6 in this study proved to be the most reliable cytokine biomarker for discriminating EOC serous patients from the control group. At a cutoff value of $75 \mathrm{pg} / \mathrm{ml}$ for IL-6, the sensitivity was $92 \%$ and the specificity was $80 \%$. Combining CA125 and IL-6 further improved specificity. In patients with serum levels above the cutoff point of CA125>35 kUI/L, a cutoff point of IL-6 $>45 \mathrm{pg} / \mathrm{ml}$ gave a specificity of $100 \%$ for distinguishing between EOC and control group (Fig. 2).

\section{Discriminating potential of ascites inflammation-regulating factors to identify women with intrinsic drug resistance} Inflammation has been associated with tumor progression and drug resistance [18, 19]. Serous EOC ascites has been previously shown to inhibit drug-induced apoptosis [20-23]. Inflammation-regulating factors may enhance cisplatin resistance $[32-35,42,44,46]$. ROC were created to determine the predictive value of ascites IL-6, IL-10, leptin, OPG, suPAR and CCL18 for discriminating, at baseline, clinically resistant patients from those that are sensitive. The clinical and pathological characteristics of the patients in our cohort are shown in Table 2. Of the 53 patients, 42 were drug sensitive and 11 were drug resistant. The median age at diagnosis was 60 years (range, 27 to 85 years), and all patients had advanced-stage (FIGO stages III/IV) with serous histology. Most ( $\geq 79 \%)$ of patients were optimally cytoreduced after initial surgery, and about $30 \%$ received pre-operative chemotherapy. There was no significant difference between the two groups. All patients had a follow-up $\geq 12$ months

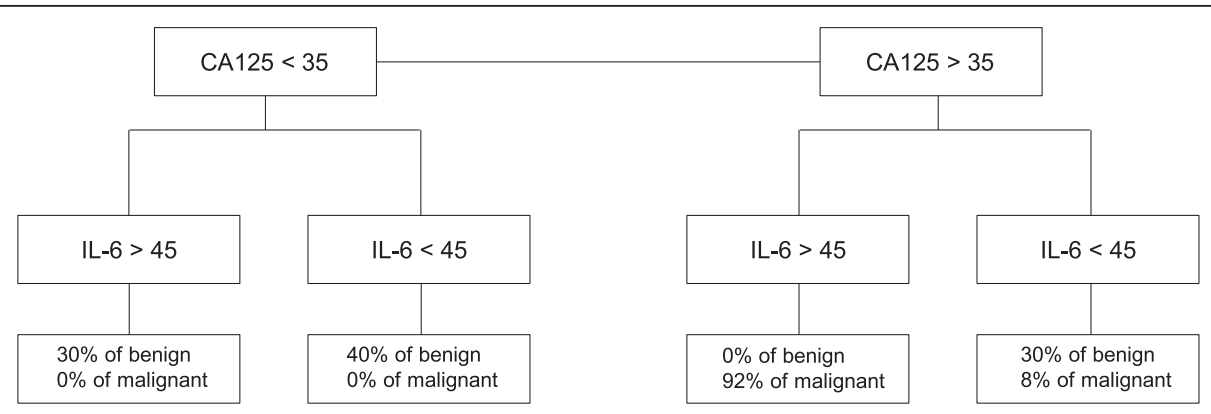

Fig. 2 Serum CA125 and ascites IL-6 levels can discriminate between patients with serous EOC or benign gynecological conditions. The markers with cutoff (pg/ml for IL-6 and kUI/L for CA125) are depicted together with the percentage of the patients with EOC or benign conditions that were predicted by the combination of markers 
Table 2 Patient characteristics

\begin{tabular}{|c|c|c|c|}
\hline Characteristic & $\begin{array}{l}\text { Drug sensitive } \\
\text { patients }\end{array}$ & $\begin{array}{l}\text { Drug resistant } \\
\text { patients }\end{array}$ & $P$ value \\
\hline$n=53$ & $(n=42)$ & $(n=11)$ & \\
\hline Age (years) & & & NS \\
\hline Median & 61,5 & 62 & \\
\hline Range & $31-81$ & $27-89$ & \\
\hline FIGO stage & & & NS \\
\hline$|-| \mid$ & $0(0)$ & $0(0)$ & \\
\hline III-IV & $42(100)$ & $11(100)$ & \\
\hline Grade & & & NS \\
\hline 1 & $4(10.5)$ & $0(0)$ & \\
\hline 2 & $8(21)$ & $2(18)$ & \\
\hline 3 & $19(50)$ & $8(73)$ & \\
\hline ND & $0(0)$ & $1(9)$ & \\
\hline $\begin{array}{l}\text { Histologic } \\
\text { subtype }\end{array}$ & & & NS \\
\hline Serous & $42(100)$ & $11(100)$ & \\
\hline Debulking status & & & NS \\
\hline$<2 \mathrm{~cm}$ & $33(79)$ & $9(82)$ & \\
\hline$>2 \mathrm{~cm}$ & $5(12)$ & $2(18)$ & \\
\hline ND & $4(10)$ & $0(0)$ & \\
\hline $\begin{array}{l}\text { Prior } \\
\text { chemotherapy }\end{array}$ & & & NS \\
\hline Yes & $9(21)$ & $4(36)$ & \\
\hline No & $33(79)$ & $7(64)$ & \\
\hline $\begin{array}{l}\text { CA125 at } \\
\text { diagnosis }\end{array}$ & & & NS \\
\hline Median & 626 & 1145 & \\
\hline Range & $20-6549$ & $88-14,180$ & \\
\hline
\end{tabular}

FIGO international federation of gynecology and obstetrics, NS not statistically significant, ND not determined

(range, 12 to 108 months). Clinically sensitive patients have a median PFS of 13.9 months and clinically resistant patients a median PFS of 4 months.

Median ascites levels of IL- 6 and IL-10, and serum levels of CA125, were not statistically different between patients that had drug sensitive or drug resistant diseases (Fig. 3a-c). Similarly, median levels of leptin, suPAR and CCL18 were not significantly different (data not shown). In contrast, ascites OPG levels were significantly higher in chemosensitive patients compared to resistant patients (Fig. 3d). ROC analysis for individual cytokines revealed low discriminating potential to stratify patients according to their sensitivity to first-line treatment (Additional file 1: Figure S1). To improve the accuracy, we assessed combinations of the studied cytokines and CA125 in ROC analysis. The combination of biomarkers with the highest discriminating potential was with CA125 and leptin (AUC $=0.936,95 \%$ CI: 0.894-
0.978) (Fig. 2d). All other combination, including CA125 with suPAR (Fig. 3d) and CA125 with IL-6 (Fig. 3e), had low discriminating potential with $\mathrm{AUC}<0.650$.

\section{Inflammation-regulating factor levels as prognostic marker in serous EOC}

We assessed the prognostic value of IL-6, IL-10, leptin, OPG, suPAR and CCL18 in relation with PFS in the cohort of 53 patients. A cutoff value corresponding to the median of each factor was used to separate patients into two groups: those with high ascites levels versus those with low ascites levels. Kaplan-Meier curves of the six factors are shown in Fig. 4. Among the six inflammationregulating factors, only IL-6 was significantly associated with a worse outcome. Patients with low ascites IL-6 levels had a median PFS of 12 months compared to patients with high levels who had a PFS of 28 months $(P=0.0004$, log rank test).

\section{Discussion}

We selected for this study patients with advanced serous EOC to ensure a homogenous group of patients and because this subtype is the most frequently encountered subtype in clinic. In this context, the conclusions of this study may not apply to other ovarian cancer sub-types or to patients presenting with FIGO stage I/II diseases. However, this study has the advantage of comprising a homogeneous group of women with advanced serous EOC, thus limiting potential bias associated with inclusion of various sub-types with distinct genetic backgrounds. In our study, ascites levels of IL-6, IL-10 and OPG were found to be elevated in patients with advanced stage serous EOC compared with patients with benign gynecological conditions. Moreover, determination of IL-6 levels could classify $68 \%$ of the advanced stage serous EOC patients accurately, without falsely classifying patients with benign gynecological conditions. These findings are in line with previous studies demonstrating higher levels of IL-6, IL-10 and OPG in malignant ascites or serum compared to patients with benign conditions $[29,47,48]$. In a recent study, IL-6 levels in ascites were the most discriminating to distinguish EOC patients from patients with benign conditions among ten selected factors [49]. Without surprise, serum CA125 levels were found to be the most discriminating factor for advanced stage serous EOC patients. Indeed, CA125 was elevated ( $>35 \mathrm{kUI} / \mathrm{L}$ ) in $100 \%$ of EOC patients and in $30 \%$ of patients with benign conditions in this study. Others found CA125 commonly elevated in serous EOC patients but it has not always consistently discriminated between malignant and benign pelvic mass [50]. Serum CA125 may be elevated in a variety of other benign conditions $[17,50]$. Therefore, CA125 alone lacks specificity. Our data suggest that ascites IL-6 might be a 


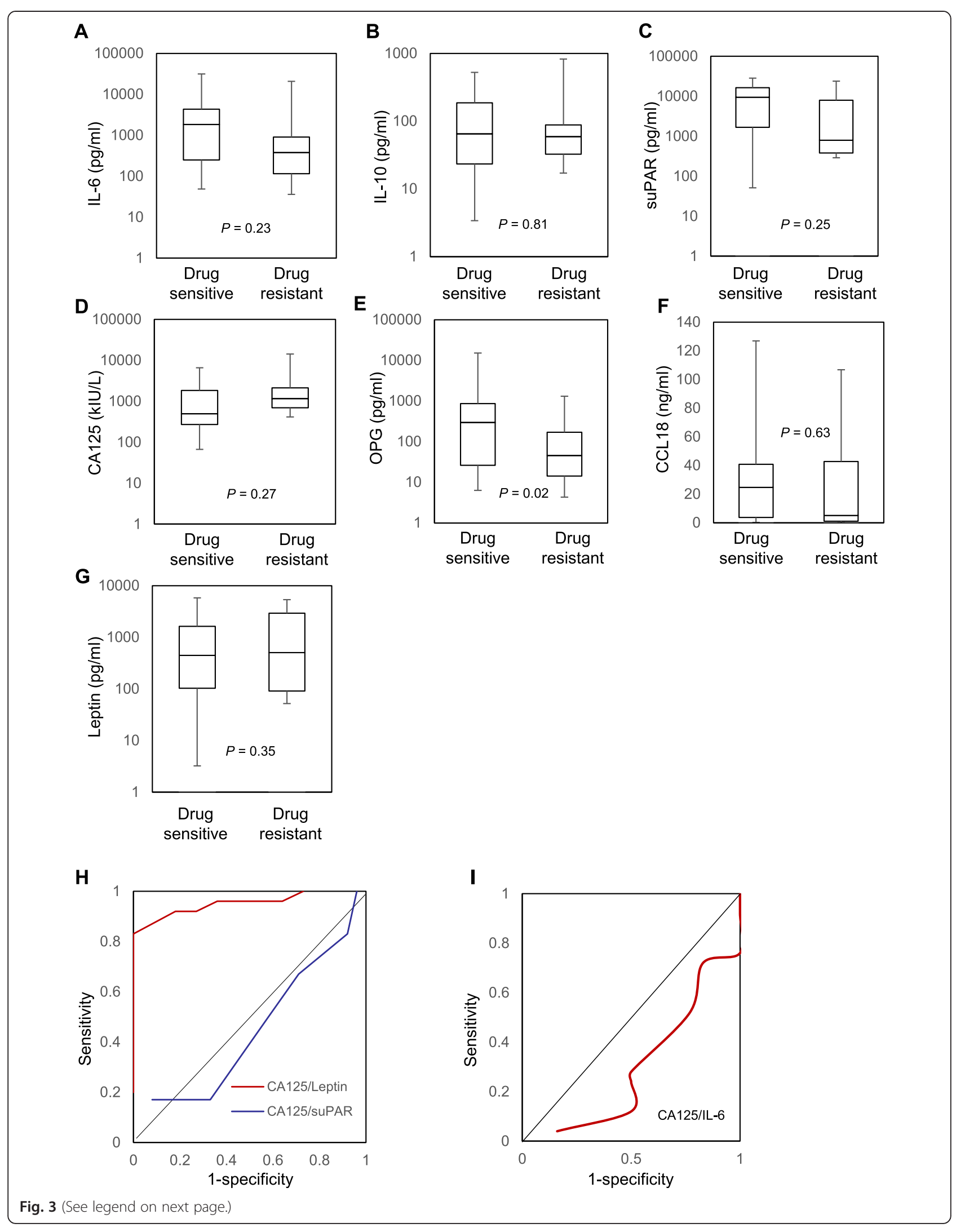


(See figure on previous page.)

Fig. 3 Ascites levels of inflammation-regulating factors in clinically resistant patients and those sensitive to first-line treatment. Box plots representing ascites levels of IL-6 (a), IL-10 (b), suPAR (c), serum CA125 (d), OPG (e), CCL18 (f) and leptin (g) in patients with resistance to first-line therapy and patients with sensitive diseases. The $P$ value is indicated for each factor. ROC analysis using the combination of CA125/leptin and CA125/suPAR (h) and CA125/IL-6 (i) for distinguishing patients with resistant or sensitive EOC

good addition to serum CA125 for diagnosis of serous EOC versus benign conditions. In our study, a cutoff point of CA125 > $35 \mathrm{kUI} / \mathrm{L}$ and a cutoff point of IL-6 > $45 \mathrm{pg} / \mathrm{ml}$ gave a sensitivity of $92 \%$ and a specificity of $100 \%$ for distinguishing between EOC and control group. One limitation of this study is that data were derived from a small number of samples, thus conclusions should be viewed appropriately. Further studies however are needed to evaluate the additional value of ascites IL-6 in combination with serum CA125 to discriminate advanced stage serous EOC patients and patients with benign gynecological conditions. Indeed, because of its retrospective nature, a confirmation of our results in a larger cohort is necessary.

IL-6 production generates an inflammatory environment that promotes metastatic growth. In this context, there is a number of studies that linked serum or ascites IL-6 levels with a worse prognosis and poor overall survival in EOC patients $[31,51,52]$. In line with these studies, our data demonstrate that higher IL-6 levels were significantly associated with shorter PFS. In addition, IL-6 has been associated, in some context, with cisplatin resistance in vitro through upregulation of anti-apoptotic proteins, such as Bcl-2 and IAPs, and downregulation of pro-apoptotic proteins, such as BID and BAX [34, 53]. In this study however, we did not observed a correlation between IL- 6 levels in ascites and clinical resistance to cisplatin. Furthermore, using IL-6 concentrations (500 to $5000 \mathrm{pg} / \mathrm{ml}$ ) at levels similar to those found in ascites, we have found no effect on cisplatin-induced cell death in EOC cell lines (data not shown). IL-6 does however promotes cell migration and invasion in vitro

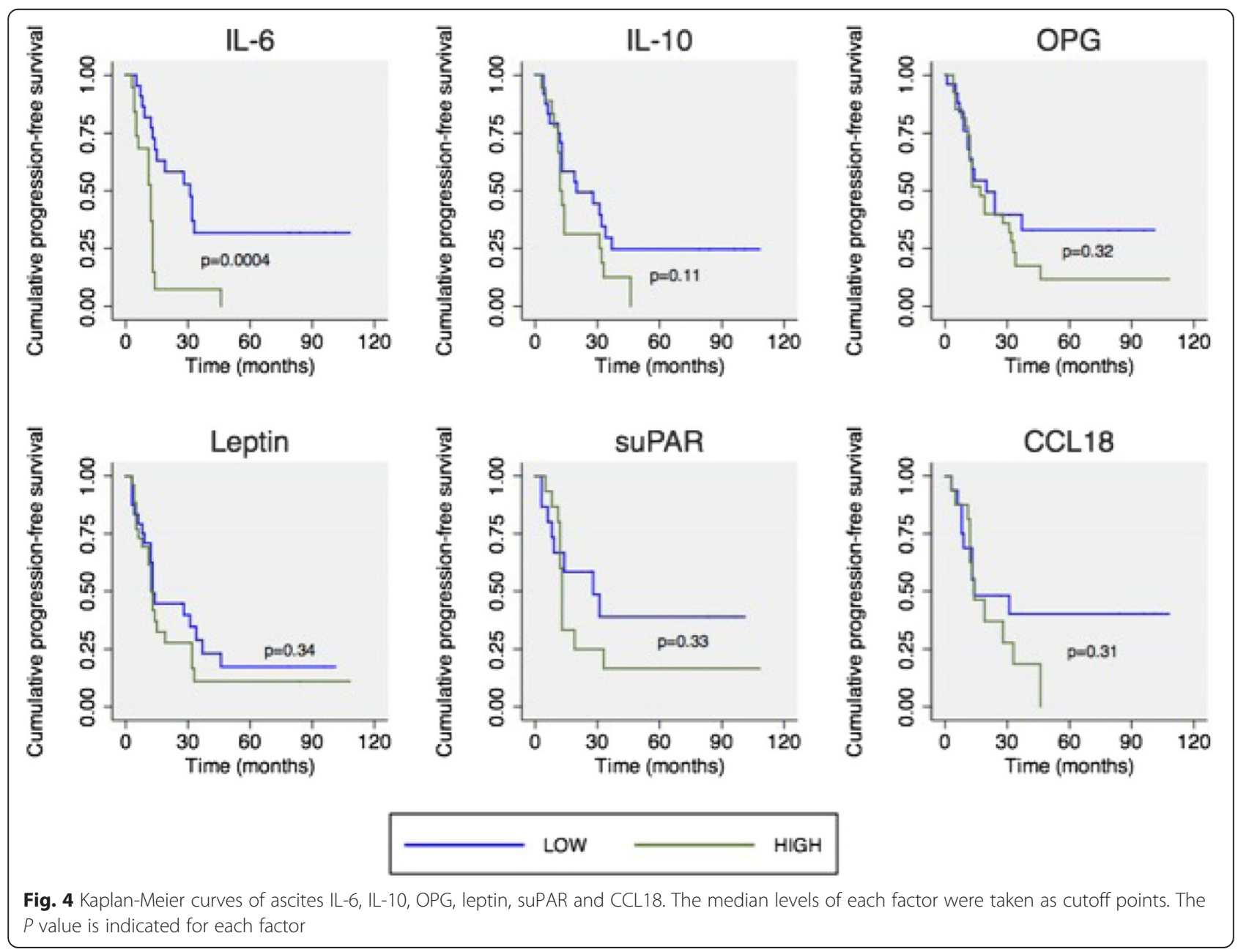


as such may contribute to metastatic growth and worse prognosis.

The second goal of the study was to determine if a single inflammation-regulating factor, or a combination of factors, could be used as a predictive value to discriminate clinically resistant versus sensitive patients. This is critical because the prognosis of women with EOC is strongly associated with the length of PFS after first-line therapy [54]. The availability of biomarkers to predict responses to the initial therapy would provide a practical approach to identify women who would benefit from a more appropriate first-line treatment. Because ascites is a proinflammatory milieu rich in cytokines, chemokines and growth factors, and because ascites may enhance resistance to various drugs, it constitutes an excellent reservoir for the identification of drug resistance biomarkers. There is a large effort in the field of EOC to identify new diagnostic and prognostic biomarkers, in particular for clinically resistant patients [55-57]. Huang et al. have performed proteomic studies of ovarian cancer ascites using gel electrophoresis coupled with matrix-assisted laser desorption/ionization time-of-flight mass spectrometry, and compared chemoresistant and chemosensitive patients [55]. They found that ceruloplasmin levels, an acute phase protein, was significantly higher in chemoresistant than in chemosensitive ascites. Such acute phase protein levels are often modulated by chemotherapy treatments [58]. Therefore, ceruloplasmin may act not as a causal protein but as a marker of systemic inflammation. In ROC analysis, the combination of CA125 and leptin had the highest discriminating potential (AUC 0.936) to distinguish clinically resistant patients to first-line therapy from sensitive patients presenting with advanced serous EOC.

Interestingly, CA125 expression has been associated with resistance to cisplatin and death receptor ligand in ovarian and breast cancer cell lines [59-61]. It was suggested that CA125 affects tumor cells by altering the expression of pro- and anti-apoptotic proteins [59, 61]. Leptin has been shown to activate PI3K/Akt and ERK1/ 2 survival pathways and stimulate the expression of antiapoptotic protein Mcl-1 in ovarian cancer cell line OVCAR3 [62]. Furthermore, serous EOC ascites was found to activate PI3K/Akt and ERK1/2 pathways and stimulate the expression of Mcl-1 in ovarian cancer cells $[20,22]$. These signaling alterations were associated with increased resistance to death receptor-induced apoptosis. Altogether, these data provide a biological rationale for the findings that the combination of CA125 and leptin discriminate between sensitive and resistant patients.

\section{Conclusions}

In conclusion, ascites IL-6 was found to be strongly related to serous EOC and may be used in combination with CA125 for diagnosis of advanced serous EOC. This finding however requires further validation. Serum CA125 in combination with leptin has the potential to discriminate clinically resistant from sensitive patients at baseline and could therefore be used to stratify patients at baseline that are more likely to benefit from standard first-line treatment among patients presenting with advanced serous EOC. The potential role of CA125 and leptin needs to be further explored.

\section{Additional file}

Additional file 1: Figure S1. Receiver operator curve (ROC) analysis by
using single inflammation-regulating factor to differentiate patients resistant
to first-line treatment (PFS $<6$ months) from those that are clinically
sensitive to first-line treatment (PFS $>6$ months).

\section{Competing interest}

The authors declare that they have no competing interests.

\section{Authors' contributions}

$\mathrm{DL}$ participated in the design of the study and performed the assays for measuring IL-6, IL-10, OPG, leptin, suPAR and CCL18 levels in ascites. IM was responsible for obtaining the ascites and the clinical data. She also performed the cytokine chip arrays experiments. AC performed the survival analyses. Pathological specimens were reviewed by PGG or CL. CR participated in the design of the study and helped to draft the manuscript. AP conceived the study, participated in its design and drafted the manuscript. All authors read and approved the final manuscript.

\section{Acknowledgments}

This work was supported by a grant from the Canadian Institutes of Health Research (A.P.), by the Centre d'excellence en Inflammation-Cancer de I'Université de Sherbrooke and by the "Programme d'aide de financement interne" of the Centre de Recherche du Centre Hospitalier Universitaire de Sherbrooke. We wish to thank the Banque de tissus et de données du Réseau de Recherche en Cancer du Fond de Recherche du Québec en Santé (FRQS), affiliated to the Canadian Tumor Repository Network (CTRNet) for providing the ascites samples.

\section{Author details}

${ }^{1}$ Département de Microbiologie et Infectiologie, Faculté de Médecine, Université de Sherbrooke, 3001, 12ième Avenue Nord, J1H 5 N4 Sherbrooke, Canada. ${ }^{2}$ Département de Pathologie, Faculté de Médecine, Université de Sherbrooke, 3001, 12ième Avenue Nord, J1H 5 N4 Sherbrooke, Canada.

Received: 16 March 2015 Accepted: 19 June 2015

Published online: 01 July 2015

References

1. Partridge EE, Barnes MN. Epithelial ovarian cancer: prevention, diagnosis, and treatment. CA Cancer J Clin. 1999;49:297-320.

2. Jemal A, Siegel R, Xu J, Ward E. Cancer Statistics 2010. CA Cancer J Clin. 2010;60:277-300

3. Bast RC, Hennessy B, Mills GB. The biology of ovarian cancer: new opportunities for translation. Nat Rev Cancer. 2009;9:415-28.

4. Ozols RF, Bookman MA, Connolly DC, Daly MB, Godwin AK, Schilder RJ, et al. Focus on epithelial ovarian cancer. Cancer Cell. 2004;5:19-24.

5. Cannistra SA. Cancer of the ovary. N Engl J Med. 2004;351:2519-29.

6. Chien J, Kuang R, Landen C, Shridhar V. Platinum-sensitive recurrence in ovarian cancer: the role of the tumor environment. Front Oncol. 2013;3:251.

7. Yin BW, Lloyd KO. Molecular cloning of the CA125 ovarian cancer antigen: identification as a new mucin (Muc16). J Biol Chem. 2001;276:27371-5.

8. Yin BW, Dnistrian A, Lloyd KO. Ovarian cancer antigen CA125 is encoded by the MUC16 mucin gene. Int J Cancer. 2002;98:737-40.

9. O'Brien TJ, Beard JB, Underwood LJ, Shigemasa K. The CA125 gene: a newly discovered extension of the glycosylated $\mathrm{N}$-terminal domain doubles the size of this extracellular superstructure. Tumour Biol. 2002;23:154-69. 
10. Canney PA, Moore M, Wilkinson PM, James RD. Ovarian cancer antigen CA125: a prospective clinical assessment of its role as a tumour marker. Br J Cancer. 1984;50:765-9.

11. Vergote IB, Bormer OP, Abeler VM. Evaluation of serum CA 125 levels in the monitoring of ovarian cancer. Am J Obstet Gynecol. 1987;157:88-92.

12. Bast Jr RC, Klug TL, St-John E, Jenison E, Niloff JM, Lazarus H, et al. A radioimmunoassay using a monoclonal antibody to monitor the course of epithelial ovarian cancer. N Engl J Med. 1983;309:883-7.

13. Rancourt C, Matte I, Lane D, Piché A. The role of MUC16 mucin (CA125) in the pathogenesis of ovarian cancer. Chapter 4 in Ovarian Cancer: Basic science perspective. INTECH open access publisher: Janeza Trdine 9, 51000 Rijeka, Croatia; 2012

14. Moore RG, Miller MC, DiSilvestro P, Landrum LM, Gajewski W, Ball JJ, et al. Evaluation of the diagnostic accuracy of the risk of ovarian malignancy algorithm in women with pelvic mass. Obstet Gynecol. 2011;118:280-8.

15. Moore RG, McMeekin DS, Brown AK, DiSilvestro P, Miller MC, Allard WJ, et al. A novel multiple marker bioassay utilizing HE4 and CA125 for the prediction of ovarian cancer in patients with pelvic mass. Gynecol Oncol. 2009;112:40-6.

16. Molina R, Escudero JM, Augé JM, Filella X, Foj L, Torné A, et al. HE4 a nove tumour marker for ovarian cancer: comparison with CA125 and ROMA algorithm in patients with genaecological diseases. Tumor Biol. 2011;32:1087-95.

17. Felder M, Kapur A, Bosquet JG, Horibata S, Heintz J, Albrecht R, et al. MUC16 (CA125): tumor biomarker to cancer therapy, work in progress. Mol Cancer. 2014;13:129.

18. Hanahan $\mathrm{H}$, Weinberg RA. Hallmarks of cancer: the next generation. Cell. 2011;144:646-74

19. Mantovani A, Allavena P, Sica A, Balkwill F. Cancer-related inflammation. Nature. 2008:454:436-44

20. Lane D, Robert V, Grondin R, Rancourt C, Piché A. Malignant ascites protect against TRAlL-induced apoptosis by activating the PI3K/Akt in human ovarian carcinoma cells. Int J Cancer. 2007;121:1227-37.

21. Lane D, Goncharenko-Khaider N, Rancourt C, Piché A. Ovarian cancer ascites protects from TRAlL-induced cell death through av $\beta 5$ integrin-mediated focal adhesion kinase and Akt activation. Oncogene. 2010;29:3519-31.

22. Goncharenko-Khaider N, Matte I, Lane D, Rancourt C, Piché A. Ovarian cancer ascites increase Mcl-1 expression in tumor cells through ERK1/2-Elk-1 signaling to attenuate TRAIL-induced apoptosis. Mol Cancer. 2012;11:84.

23. Lane D, Matte I, Rancourt C, Piché A. The prosurvival activity of ascites against TRAIL is associated with a shorter disease-free interval in patients with ovarian cancer. J Ovarian Res. 2010;3:1.

24. Mills GB, May C, McGill M, Roifman CM, Mellors A. A putative new growth factor in ascitic fluid from ovarian cancer patients: identification, characterization, and mechanism of action. Cancer Res. 1988;48:1066-71.

25. Mills GB, May C, Hill M, Campbell S, Shaw P, Marks A. Ascitic fluid from human ovarian cancer patients contains growth factors necessary for intraperitoneal growth of human ovarian adenocarcinoma cells. J Clin Invest. 1990:86:851-5.

26. Richardson M, Gunawan J, Hatton MW, Seidlitz E, Hirte HW, Singh G. Malignant ascites fluids (MAF), including ovarian cancer-associated MAF, contains angiostatin and other factor(s) which inhibit angiogenesis. Gynecol Oncol. 2002;86:279-87.

27. Xu Y, Gaudette DC, Boynton JD, Frankel A, Fang XJ, Sharma A, et al. Characterization of an ovarian cancer activating factor in ascites of ovarian cancer patients. Clin Cancer Res. 1995;1:1223-32.

28. Yamada T, Sato K, Komachi M, Malchinkhuu E, Tobo M, Kimura T, et al. Lysophosphatidic acid (LPA) in malignant ascites stimulates motility of human pancreatic cancer cells through LPA1. J Biol Chem. 2004;279:6595-605.

29. Giuntoli RL, Webb TJ, Zoso A, Rogers O, Diaz-Montes TP, Bristow RE, et al. Ovarian cancer-associated ascites demonstrates altered immune environment: implications for antitumor immunity. Anticancer Res. 2009;29:2875-84.

30. Matte I, Lane D, Laplante C, Rancourt C, Piché A. Profiling of cytokines in human epithelial ovarian cancer ascites. Am J Cancer Res. 2012;2:566-80

31. Lane D, Matte I, Piché A. Prognostic significance of IL-6 and IL-8 ascites levels in ovarian cancer patients. BMC Cancer. 2011;11:210.

32. Shahzad MM, Arevalo JM, Armaiz-Pena GN, Lu C, Stone RL, Moreno-Smith $M$, et al. Stress effects on FosB- and interleukin-8 (IL8)-driven ovarian cancer growth and metastasis. J Biol Chem. 2010;285:35462-70.

33. Johnson MT, Gotlieb WH, Rabbi M, Martinez-Maza O, Berek JS. Induction of cisplatin resistance and metallothionein expression by interleukin-6. Gynecol Oncol. 1993;49:110.
34. Cohen S, Bruuchim I, Graiver D, Evron Z, Oron-Karni V, Pasmanik-Chor M, et al. Platinum-resistance in ovarian cancer cells is mediated by IL-6 secretion via the increased expression of its target ClAP-2. J Mol Med. 2013:91:357-68.

35. Coward II, Kulbe H. The role of interleukin-6 in gynaecological malignancies. Cytokine Growth Factor Rev. 2012;23:333-42

36. Hanash SM, Pitteri S, Faca VM. Mining the plasma proteome for cancer biomarker. Nature. 2008:452:571-9.

37. Rustin GJ, Timmers P, Nelstrop A, Shreeves G, Bentzen SM, Baron B, et al. Comparison of CA-125 and standard definitions of progression of ovarian cancer in the intergroup trial of cisplatin and paclitaxel versus cisplatin and cyclophosphamide. J Clin Oncol. 2006;24:45-51.

38. Lane D, Matte I, Rancourt C, Piché A. Osteoprotegerin (OPG) protects ovarian cancer cells from TRAlL-induced apoptosis but does not contribute to malignant ascites-mediated attenuation of TRAlL-induced apoptosis. J Ovarian Res. 2012;5:34.

39. Lane D, Matte I, Laplante C, Garde-Granger P, Rancourt C, Piché A Osteoprotegerin (OPG) activates integrin, focal adhesion knase (FAK) and Akt signaling in ovarian cancer cells to attenuate TRAIL-induced apoptosis. J Ovarian Res. 2013;6:82.

40. Duan Z, Foster R, Bell DA, Mahoney J, Wolak K, Vaidya A, et al. Signal transducers and activators of transcription 3 pathway activation in drug-resistant ovarian cancer. Clin Cancer Res. 2006:12:5055-63.

41. Guo Y, Nemeth J, O'Brien C, Susa M, Liu X, Zhang Z, et al. Effects of siltuximab on the IL-6-induced signaling pathway in ovarian cancer. Clin Cancer Res. 2010;16:5759-69.

42. $Y$ an $H Q$, Huang $X B$, Ke SZ, Jiang $Y N$, Zhang $Y H$, Wang $Y N$, et al. Interleukin-6 augments lung cancer chemotherapeutic resistance via ataxia-telangiectasia mutated/NF-kappaB pathway activation. Cancer Sci. 2014;105:1220-7.

43. Zeng L, O'Connor C, Zhang J, Kaplan AM, Cohen DA. IL-10 promotes resistance to apoptosis and metastatic potential in tumor lung tumor cell lines. Cytokine. 2010:49:294-302

44. Efferth T, Fabry U, Osieka R. Leptin contributes to the protection of human leukemic cells from cisplatinum cytotoxicity. Anticancer Res. 2000;20:2541-6.

45. Alfano D, laccarino I, Stoppelli MP. Urokinase signaling through its receptor protects against anoikis by increasing BCl-xL expression levels. J Biol Chem. 2006;281:17758-67.

46. Gutova M, Najbauer J, Gevorgyan A, Metz MZ, Weng Y, Shih CC, et al. Identification of UPAR-positive chemoresistant cells in small cell lung cancer. PLOS ONE. 2007;2:e243.

47. Lambeck AJ, Crijns AP, Leffers N, Sluiter WJ, ten Hoor KA, Braid M, et al. Serum cytokine profiling as a diagnostic and prognostic tool in ovarian cancer: a potential role for interleukin-7. Clin Cancer Res. 2007;13:2385-91.

48. Mustea A, Konsgen D, Braicu El, Pirvulescu C, Sun P, Sofroni D, et al. Expression of IL-10 in patients with ovarian carcinoma. Anticancer Res. 2006;26:1715-8.

49. Chudecka-Glaz AM, Cymbaluk-Ploska AA, Menkiszak JL, Pius-Sadowska E, Machalinski BB, Sompolska-Rzechula A, et al. Assessment of selected cytokines, proteins, and growth factors in the peritoneal fluid of patients with ovarian cancer and benign gynecological conditions. OncoTargets Ther. 2015;8:471-85.

50. Einhorn N, Knapp RC. Bast RC, Zurawski Jr VR. CA125 assay used in conjunction with CA 15-3 and TAG-72 assays for discrimination between malignant and non-malignant diseases of the ovary. Acta Oncol. 1989;28:655-7.

51. Scambia G, Testa U, Panici PB, Foti E, Martucci R, Gadducci A, et al. Prognostic significance of IL-6 serum levels in patients with ovarian cancer Br J Cancer. 1995;71:354-64.

52. Plante M, Rubin SC, Wong GY, Federici MG, Finstad CL, Gastl GA. Interleukin-6 level in serum and ascites as a prognostic factor in patients with epithelial ovarian cancer. Cancer. 1994;73:1882-8.

53. Borsellino N, Belldegrun A, Bonavida B. Endogenous interleukin-6 is a resistance factor for cis-diamminedichloroplatinum and etoposide-mediated cytotoxicity of human prostate carcinoma cell lines. Cancer Res. 1995;55:4633-9.

54. Spriggs D. Optimal sequencing in the treatment of recurrent ovarian cancer Gynecol Oncol. 2003;90:539-44

55. Huang H, Li Y, Liu J, Zheng M, Feng Y, Hu K, et al. Screening and identification of biomarkers in ascites related to intrinsic chemoresistance of serous epithelial ovarian cancers. PLoS ONE. 2012;7:51256.

56. Han Y, Huang H, Xiao Z, Zhang W, Cao Y, Qu L, et al. Integrated analysis of gene expression profiles associated with response of platinum/paclitaxel-based treatment in epithelial ovarian cancer. PLoS ONE. 2012;7:52745. 
57. Bachvarov D, L'Esperance S, Popa I, Bachvarova M, Plante M, Têtu B. Gene expression patterns of chemoresistant and chemosenstive serous epithelial ovarian tumors with possible predictive value in response to initial chemotherapy. Int J Oncol. 2006;29:919-33.

58. Weijl NI, Hopman GD, Wipkink-Bakker A, Lentjes EG, Berger HM, Cleton FJ, et al. Cisplatin combination chemotherapy induces a fall in plasma antioxidants of cancer patients. Ann Oncol. 1998:9:1331-7.

59. Lakshmanan I, Ponnusamy MP, Das S, Chakraborty S, Haridas D, Mukhopadhyay $P$, et al. MUC16 induced rapid G2/M transition via interactions with JAK2 for increased proliferation and anti-apoptosis in breast cancer cells. Oncogene. 2012;31:805-17.

60. Matte I, Lane D, Boivin M, Rancourt C, Piché A. MUC16 mucin (CA125) attenuates TRAIL-induced apoptosis by decreasing TRAIL receptor R2 expression and increasing c-FLIP expression. BMC Cancer. 2014;14:234

61. Boivin M, Lane D, Rancourt C, Piché A. CA125 (MUC16) tumor antigen selectively modulates the sensitivity of ovarian cancer cells to genotoxic drug-induced apoptosis. Gynecol Oncol. 2009:115:407-13.

62. Chen C, Chang YC, Lan MS, Breslin M. Leptin stimulates ovarian cancer cell growth and inhibits apoptosis by incresing cyclin D1 and Mcl-1 expression via the activation of the MEK/ERK1/2 and PI3K/Akt signaling pathways. Int J Oncol. 2013;42:1113-9.

\section{Submit your next manuscript to BioMed Central and take full advantage of:}

- Convenient online submission

- Thorough peer review

- No space constraints or color figure charges

- Immediate publication on acceptance

- Inclusion in PubMed, CAS, Scopus and Google Scholar

- Research which is freely available for redistribution 